\title{
Procesos de obtención y evaluación de aleaciones preciosas base oro usadas en joyería
}

\author{
Luz Amparo Quintero Ortiz \\ Universidad Industrial de \\ Santander \\ luzquint@uis.edu.co
}

\author{
Mayerly Jhojana Fernández \\ Torres \\ Universidad Industrial de Santander \\ maye0826@gmail.com
}

\author{
Arnaldo Alonso Baquero \\ Universidad Industrial de \\ Santander \\ arnaldo@uis.edu.co
}

(Tipo de Artículo: Investigación. Recibido el 17/04/2015. Aprobado el 19/07/2015)

Resumen. Los primeros estudios realizados sobre aleaciones base oro se desarrollaron hace algunas décadas, los cuales hacen referencia a los proceso de fabricación de piezas de diferentes tamaños y formas e incluyen problemas debidos a reacciones alergias generados por su uso continuo. Con el paso del tiempo, los nuevos desarrollos tecnológicos y el surgimiento de técnicas de estudio y caracterización, se han realizado avances que permiten dar una posible explicación al comportamiento del material, el cual tiene una íntima relación con su microestructura y sus propiedades. Además se han podido fabricar aleaciones con composiciones predeterminadas, tanto cristalinas como amorfas, así como aplicar diferentes procesos de fabricación y caracterización.

Palabras clave. Aleaciones de oro, fundición, joyería.

\section{Obtaining processes and test precious alloys gold used in jewellery}

\begin{abstract}
First studies made about golden alloys are developed some decades ago, which refer to the manufacturing process pieces of different sizes and shapes and include problems due allergies reactions generated for continuous use. Over time, new technological development and the emergence of study and characterization technical, there has competed advances that allow give a possible explanation to material behavior, which has a close relationship their microstructure and properties. Furthermore, it has been possible to make alloys with predetermined composition, crystalline and amorphous, applying manufacturing process and characterization.
\end{abstract}

Keywords. Casting, gold alloys, jewellery.

\section{INTRODUCCIÓN}

La joyería posee un gran número de variables que necesitan ser monitoreadas durante la producción de las piezas, para garantizar su calidad y las características y propiedades necesarias. Entre estas variables se encuentran la selección de los elementos de aleación, la elaboración de modelos y moldes, el proceso de fusión y las condiciones de operación.

Aunque la experiencia ganada en estos procesos, hace aportes significativos para el logro de un buen resultado, en la actualidad, la modernización en los equipos para fusión de los materiales, el uso de software para diseño de modelos y la disponibilidad de información termodinámica amplia sobre aleaciones y materiales, permite optimizar los procesos, con mejor control y garantía de resultados exitosos.

Por otra parte, es muy útil el conocimiento y uso de técnicas de caracterización y evaluación de las aleaciones con el fin de determinar su posible comportamiento frente a la aplicación para la cual fue diseñada.

En el presente estudio se hace una revisión bibliográfica de aspectos fundamentales asociados con las aleaciones preciosas base oro, como son: los procesos de fabricación y las múltiples variables que lo afectan y las técnicas de caracterización y evaluación de sus propiedades físicas y químicas. Se hace mención a software de apoyo que contribuye en la selección de materias primas y en la simulación del proceso de colada, de gran importancia para la producción de una pieza de joyería de manera más eficiente y controlada. Finalmente se hace referencia a trabajos futuros en el área, derivados de la revisión hecha.

\section{PROCESOS DE FABRICACIÓN}

En la fabricación de una aleación es importante tener en cuenta las características de las materias primas, las propiedades metalúrgicas, los procesos de fundición, el acabado superficial.

\subsection{Materias primas}

A la hora de escoger el tipo de aleación para una pieza de joyería, se deben tener en cuenta factores como sus propiedades mecánicas (resistencia y dureza), ópticas (color y brillo), colabilidad, trabajabilidad y quilate, las cuales dependen de la composición de la aleación y de la estructura de la misma [1]. Un aspecto determinante que permite obtener una aleación preciosa base oro, con las propiedades deseadas, es la selección adecuada de los elementos de aleación. Sin embargo, la elección de los mismos dependen no solo de los factores mencionados, sino también de otros que están interrelacionados, estos son: la solubilidad de los elementos, las fases presentes, la presencia de 
compuestos intermetálicos y el punto fusión de las especies, entre otros.

Una herramienta de apoyo que permite correlacionar estos factores son los diagramas de equilibrio. Estos son mapas termodinámicos que contienen información acerca de la composición de la aleación con la temperatura [2].

A partir de ellos es posible determinar las composiciones más adecuadas para obtener la aleación en estado líquido o conocer las fases presentes a una determinada temperatura y con esto estimar las propiedades mecánicas de la misma.

Los diagramas de fase empleados en aleaciones preciosas son multicomponentes, uno muy utilizado es el diagrama ternario, el cual se representa espacialmente como un triángulo donde las composiciones de los componentes se encuentran en cada uno de los vértices y la composición del líquido está representada por un punto en el triángulo [3].

Dichos diagramas, para las aleaciones preciosas base oro, están constituidos en muchas oportunidades principalmente por $\mathrm{Au}, \mathrm{Ag}$ y $\mathrm{Cu}$. A continuación, en la Fig. 1, se muestra el diagrama Au$\mathrm{Ag}-\mathrm{Cu}$ a una isoterma de $300^{\circ} \mathrm{C}$ [4], en el cual se observan las diferentes fases presentes a una composición determinada.

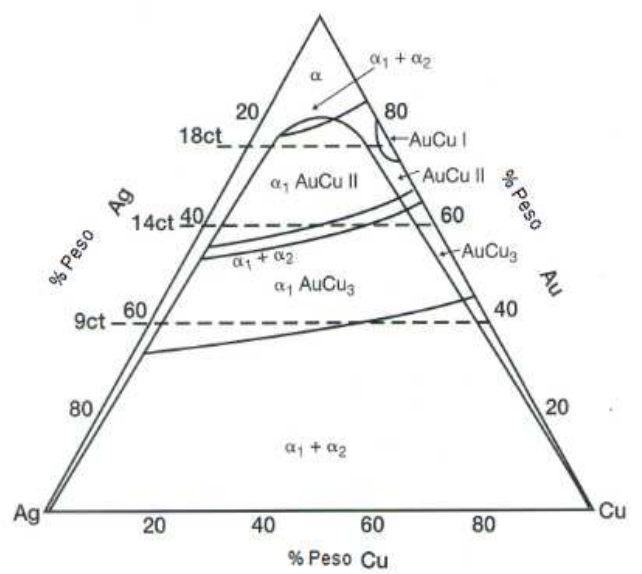

Fig. 1. Diagrama de fase $\mathrm{Au}-\mathrm{Ag}-\mathrm{Cu}$ a $300^{\circ} \mathrm{C}$ [4]

Además, se debe tener en cuenta que las aleaciones preciosas base oro, se pueden elaborar de diferentes colores tales como: blanco, amarillo, rosado, verde, entre otros. Por ello, resulta aún más útil emplear los diagramas, debido a que se pueden obtener aleaciones con un color específico, teniendo como guía para la aproximación del color la composición de la aleación. En la Fig. 2, se muestran los diferentes colores en zonas de composición, por ejemplo el color amarillo se presenta desde $100 \% \mathrm{Au}$ y $0 \%$ Cu-Ag, hasta una aleación con $25 \%$ Au, $25 \% \mathrm{Cu}$ y $50 \mathrm{Ag}$, aproximadamente.

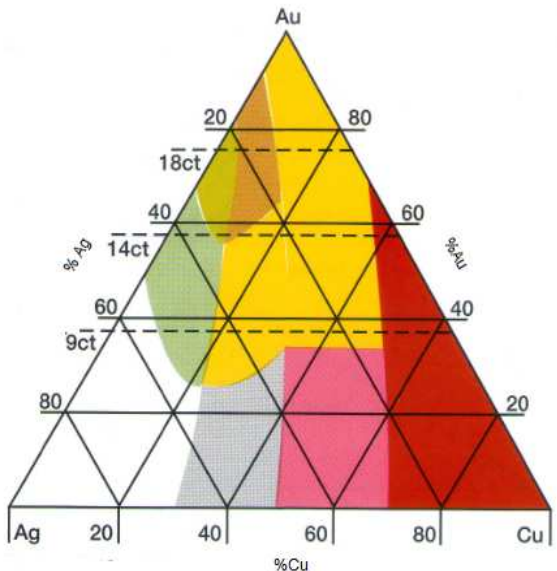

Fig. 2. Diferentes colores para las aleaciones Au-Ag$\mathrm{Cu}$ [4]

En la actualidad hay softwares de simulación, como el Thermo-Calc y FactSage, entre otros, los cuales apoyan la construcción de los diagramas ternarios, tomando como base una gran cantidad de información termodinámica disponible y ecuaciones que relacionan esta información y permiten predecir de forma teórica el comportamiento de elementos, compuestos y aleaciones.

Otros de los aspectos que se deben tener en cuenta en la selección de los elementos de aleación es su toxicidad. Este es un tema de gran importancia en la joyería pues las piezas fabricadas van a ser utilizadas por seres humanos.

En este sentido, el oro puro puede generar problemas alérgicos asociado con uso continuo de accesorios en contacto íntimo con la piel, como es el caso de los piercing y accesorios de joyería. Estas reacciones han hecho que en algunos países el oro ocupe un segundo lugar después del níquel en generar reacciones alérgicas [5].

En la Tabla 1, se encuentran otros elementos que son utilizados para la fabricación de las aleaciones base oro, pero que a su vez, generan reacciones negativas para las personas.

TABLA 1

Toxicidad de algunos elementos usados en las aleaciones base oro

\begin{tabular}{ll}
\hline Elemento & Toxicidad \\
\hline $\mathrm{Ni}$ & $\begin{array}{l}\text { La Directiva europea UNE-UN 1811 y 12472, nos } \\
\text { muestran que causa reacciones alérgicas desde } \\
\text { una pequeña irritación hasta un eczema severo }\end{array}$ \\
& $\begin{array}{l}\text { [6], [7], por tanto la velocidad de liberación debe } \\
\text { ser menor que } 5 \mu \mathrm{g} / \mathrm{cm} 2 / \text { semana [8], [9]. }\end{array}$ \\
$\mathrm{Sn}, \mathrm{Cr}, \mathrm{Mn}$, & Tóxico por inhalación [6], [10]. \\
$\mathrm{Co}$ & Tóxico, su acumulación conlleva a problemas \\
$\mathrm{Fe}$ & cancerígenos [6].
\end{tabular}

Otro factor importante que influye en el proceso de fabricación de estas aleaciones, el cual permite que 
la fundición se realice de forma óptima son los fundentes [11], los cuales son usados para prevenir la oxidación, reaccionar con algún óxido en la fundición y formar escoria en la superficie del metal.

En este caso, es importante utilizar la mínima cantidad posible, porque en ocasiones parte del fundente queda atrapado dentro de la fundición [12], generando defectos en la pieza fundida. En la Tabla 2 , se encuentran algunos de los fundentes más usados para aleaciones de oro.

TABLA 2

Fundentes típicos usados en aleaciones de oro [13]

\begin{tabular}{lll}
\hline Material & $\begin{array}{l}\text { Temperatura } \\
\text { Fusión }{ }^{\circ} \mathrm{C}\end{array}$ & Observaciones \\
\hline Bórax & $561 / 760$ & $\begin{array}{l}\mathrm{Na}_{2} \mathrm{~B}_{4} \mathrm{O}_{7} .10 \mathrm{H}_{2} \mathrm{O} \text {, disuelve } \\
\text { óxidos de cobre y cinc }\end{array}$ \\
$\begin{array}{l}0.0 \quad \text { Ácido } \\
\text { bórico }\end{array}$ & $185 / 870$ & $\begin{array}{l}\mathrm{H}_{3} \mathrm{BO}_{3} \text {, adecuado para el } \\
\text { oro blanco }\end{array}$ \\
$\begin{array}{l}\text { Carbonato de } \\
\text { sodio }\end{array}$ & 852 & $\mathrm{Na}_{2} \mathrm{CO}_{3}$, fundente básico. \\
\begin{tabular}{l} 
Sal \\
$\begin{array}{l}\text { amoniacal } \\
\text { Nitrato de } \\
\text { potasio } \\
\text { Sal común }\end{array}$ \\
\hline
\end{tabular} & 840 & $\mathrm{NH}_{4} \mathrm{Cl}$, volatiliza la escoria. \\
\hline
\end{tabular}

\subsection{Propiedades metalúrgicas}

Dentro de estas propiedades se encuentra la microestructura.

\subsubsection{Microestructura}

La microestructura de una aleación está asociada con proceso de solidificación, su composición, tratamiento térmico aplicado, el trabajado mecánico y la velocidad de enfriamiento.

El proceso de solidificación, consta de dos etapas: nucleación y crecimiento de los núcleos. La primera etapa consiste en la formación de partículas de solido iniciales por la acumulación de átomos alrededor de las impurezas o de la pared del molde, a la temperatura de fusión. También se pueden formar por las fluctuaciones térmicas en el metal. Los núcleos crecen con una dirección preferencial en forma de cristales, generando un árbol llamado dendrita. Este es el proceso más común por el cual los metales solidifican [14]. Consecuentemente, el crecimiento de las dendritas hacia el exterior de diferentes núcleos genera un contacto con cada uno, llamado límite de grano.

El tamaño de grano es controlado por el número de núcleos y por la velocidad de enfriamiento. Si solo algunos núcleos son producidos, esos pueden crecer sin obstáculos para dar un tamaño de grano relativamente grande. Como consecuencia, el material será frágil, duro y con poca trabajabilidad. Sin embargo, con una velocidad de enfriamiento alta, muchos más núcleos son producidos esto se debe a que no todos los núcleos se forman al mismo tiempo.
Como resultado el tamaño de grano es relativamente pequeño, con ello se dan las mejores propiedades como resistencia, ductilidad y dureza.

La composición de la aleación determina las fases presentes en la misma, así como la presencia de impurezas. Si estas impurezas son insolubles, tienden a ser atrapadas en la última parte del líquido a solidificar y frecuentemente forma una película continúa de material frágil entre la dendrita y en el límite de grano. Si se presentan en cantidades suficientes, debilitan la fundición y la vuelven frágil.

En ocasiones, las propiedades generadas por el proceso de fundición no son suficientes, por ello es necesario realizarle un conformado y algunos tratamientos térmicos a las piezas.

El objetivo de realizar un conformado a metales es destruir la estructura original de fundición y producir una nueva. El conformado puede ser realizado simultáneamente con el recocido, en este caso es llamado trabajo en caliente, o realizado a temperatura baja llamado trabajado en frio [15].

Por otra parte, para elaborar aleaciones no cristalinas, se utilizan velocidades de enfriamiento muy altas, por ejemplo alrededor de $10^{6} \mathrm{~K} / \mathrm{s}$, con ellas se generan las llamados vidrio metálicos. Ellos no poseen una estructura cristalina de largo alcance y pueden ofrecer excelentes propiedades para diferentes aplicaciones [16]. En este sentido, se han reportado investigaciones en las cuales se obtienen aleaciones para aplicación en la joyería, incluyendo oro de 18 quilates [17], las cuales han llegado a presentar mejores propiedades que las mismas siendo cristalinas.

Un investigador destacado en el campo de las aleaciones amorfas, Akihisa Inoue, estableció unas reglas empíricas con el fin de poder elegir los elementos que pueden formar una aleación vítrea, estos son: la aleación debe tener más de tres componentes, la diferencia en tamaños atómicos de los elementos debe estar por encima del $12 \%$ y deben tener calores negativos de mezcla.

La principal limitante en la fabricación de estas aleaciones fue el reducido espesor en que se pudieron fabricar inicialmente, siendo éste solo de unas cuantas micras. Posteriores estudios han logrado generar aleaciones vítreas con velocidades de enfriamiento menores, como es el caso de los vidrios metálicos gruesos, a través de la obtención de aleaciones multicomponentes, pudiéndose obtener en espesores mayores.

\subsection{Proceso de fundición}

El proceso de fundición se realiza teniendo en cuenta si la aleación es cristalina o amorfa. Algunos de los factores principales para los dos casos y que 
permiten una fundición óptima son el tipo de horno, crisoles, método de fundición.

\subsubsection{Hornos}

Para obtener piezas de joyería con estructura cristalina, existen diversas formas de fabricarlas, desde el uso de un simple soplete, hasta una moderna planta de fusión por inducción. Si es amorfa, es necesario disponer de mecanismos que permitan hacer el enfriamiento con una alta velocidad.

De los hornos que se encuentran en el mercado el que genera una mejor pieza fundida es el de inducción, debido a que proporciona homogeneidad en la aleación por poseer agitación electromagnética [18].

Es importante poder disminuir la oxidación generada por los gases de la atmósfera circundante, debido a que la fundición puede disolver gases como oxígeno e hidrógeno, los cuales generan defectos en la pieza fundida. Por lo anterior es recomendable usar vacío en el momento de la fundición, o un gas inerte, independientemente de si se trata de una aleación cristalina o amorfa.

\subsubsection{Crisoles}

También se considera factor importante, el crisol que se usa en la fundición, el cual puede generar contaminación de la aleación en proceso. Una de las principales características que debe tener un crisol, además de no reaccionar con los elementos que componen la aleación, es poseer una alta resistencia a la formación de grietas por choque térmico [19].

Los crisoles más usados son los de grafito para aleaciones de oro, plata y oro coloreado, debido a que se pueden usar varias veces, y presentan poca contracción. Adicionalmente contribuye en la homogenización de la temperatura del baño.

Además, existen crisoles refractarios, los cuales se usan para aleaciones de oro blanco que contengan níquel porque éste reacciona con el carbono.

En el caso de enfriamiento rápido el crisol que se usa es el de cobre por su alta conductividad térmica.

\subsection{Método de fundición}

La técnica de la cera perdida es la más utilizada para fabricar piezas en oro. Esta consiste en la fabricación de un modelo en cera, el cual es cubierto por un material refractario, la cera se funde y es extraída del refractario con el fin de generar un espacio vacío, el cual es posteriormente ocupado por la aleación fundida.

Sin embargo, esta técnica no ha sido posible implementarla con los vidrios metálicos debido al tipo de crisol usado en la colada, ya que para generar un vidrio es necesario un crisol metálico, mientras que los usados en la técnica de la cera perdida son cerámicos [20].

A continuación se muestran los diferentes pasos a seguir para la realización de la pieza fundida empleando la técnica de la cera perdida.

\subsubsection{Diseño del modelo}

El diseño del modelo puede ser realizado en papel, sin embargo con el paso del tiempo es posible diseñarlo en CAD (Computer Aided Design), mediante un software y así poder obtener una representación en $3 \mathrm{D}$, de las dimensiones reales del mismo [21], [22]. En la Fig. 3, se muestra la imagen en 3D de un anillo diseñado en un software con aplicación en joyería.

Las principales ventajas del uso de estas herramientas informáticas son: la precisión del diseño, la facilidad de almacenamiento y posibilidad de modificación en cualquier momento. Además las propiedades físicas como masa y volumen pueden ser calculados por este medio [23], [24].

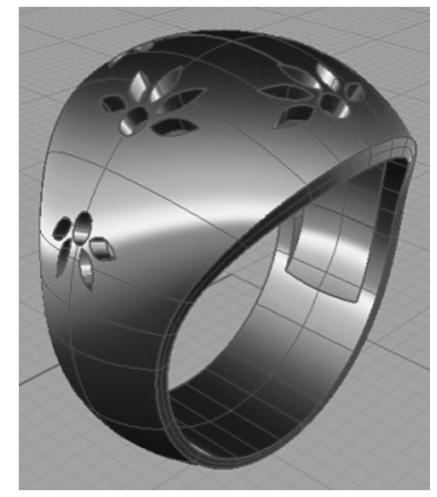

Fig. 3. Anillo diseñado en CAD

\subsubsection{Modelo en cera}

- El modelo en cera puede realizarse de dos formas, de manera manual por medio de un operario que tenga la destreza necesaria, o si se tiene el diseño en CAD realizarlo en un equipo de prototipado rápido [25]. En la Fig. 4 se muestra la imagen de un modelo en cera de un anillo.

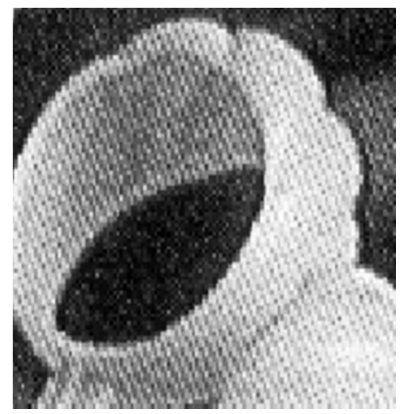

- Fig. 4. Modelo en cera [26] 


\subsubsection{Molde de goma}

Si se desea fabricar el mismo modelo en forma repetitiva, lo más conveniente es usar un molde el caucho o goma que debe tener la forma del modelo a repetir, el cual será inyectado con cera líquida el número de veces que se desee.

Una forma de fabricar el molde de goma, es obtener un modelo elaborado en metal, con buen acabado superficial, con el fin de garantizar un óptimo copiado de la pieza, además debe incluir el alimentador. Posterior a esto, se coloca el modelo en una caja metálica cuyo fondo contiene una resina, que se lleva a una temperatura entre $145-175^{\circ} \mathrm{C}$, durante un tiempo de 40 a 80 minutos, este procedimiento es llamado vulcanización [27]. En la figura 5 se puede observar el molde en goma.

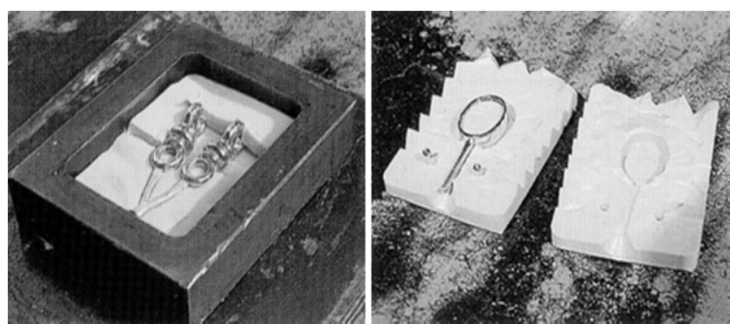

Fig. 5. Molde en goma del modelo [26]

\subsubsection{Generación del árbol de cera}

Después de general el molde en goma, se realiza la inyección de cera en el mismo, las veces que se quiera duplicar. Los modelos en cera se colocan en un alimentador principal como muestra la fig. 6 .
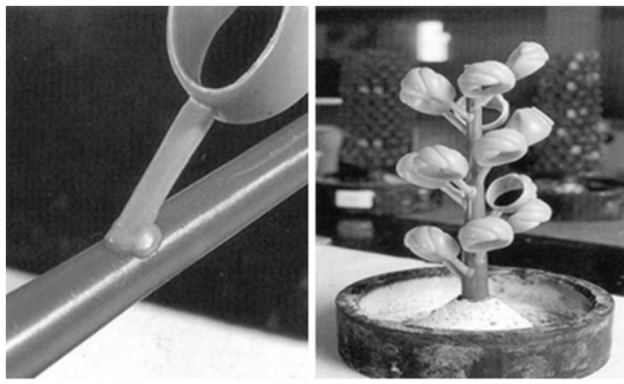

Fig. 6. Generación del árbol [26]

\subsubsection{Fabricación y tratamiento térmico de revestimiento}

El revestimiento es un material refractario cuya composición es aproximadamente de $25-30 \%$ de sulfato de calcio hemi-hidratado, el resto es cristobalita. Esta composición puede variar dependiendo del fabricante. El revestimiento se adquiere en polvo y es mezclado en proporciones recomendadas de agua a una temperatura entre 21 y $24^{\circ} \mathrm{C}$. Generalmente $1 \mathrm{~kg}$ de revestimiento contiene 0.4 litros de agua. Este es mezclado de 2 a 4 minutos en un equipo al vacío. Luego es vaciado en un cilindro en acero, el cual contiene el árbol con los diseños a fundir. Después de haber vaciado el revestimiento en el cilindro, se le realiza de nuevo vacío durante 30 segundos con el fin de evitar porosidad [28].

Posterior a la generación del revestimiento, se le realiza un tratamiento térmico similar al mostrado en la Fig. 7. En este se observan las rampas de calentamiento y sostenimiento realizadas con el fin de generar las transformaciones de fase necesarias para obtener un buen copiado de la pieza [29].

En el revestimiento la calidad del agua y el control de la temperatura son dos variables que afectan las propiedades del mismo.

Una de las recomendaciones que se debe tener en cuenta es la utilización de agua desionizada con el fin de evitar la contaminación, además controlar la velocidad de calentamiento que influye en la presencia de fases inestables y la consecuente fragilidad en el material [31].

Los revestimientos utilizados dependen del tipo de aleaciones a fundir, en la Tabla 3 , se encuentran algunas de las características esperadas en él [32].

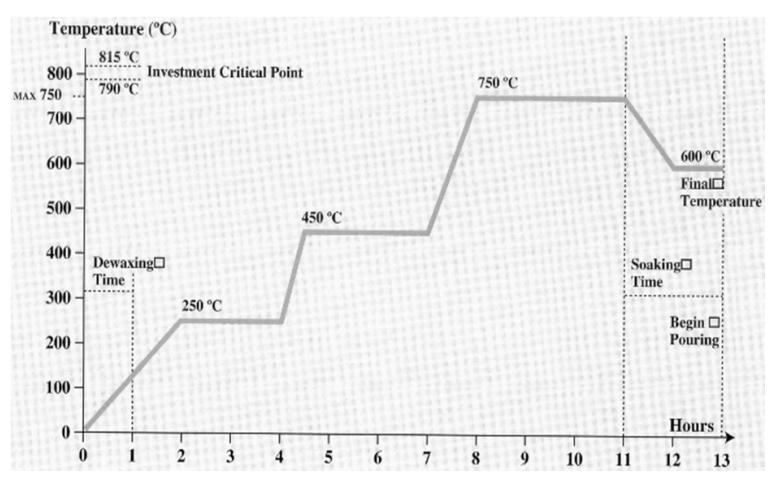

Fig. 7. Tratamiento térmico del revestimiento [30]

TABLA 3

Características del revestimiento para fundir oro [32]

\begin{tabular}{|c|c|c|c|c|}
\hline Aplicación & $\begin{array}{l}\text { Pureza } \\
\text { material }\end{array}$ & $\begin{array}{l}\text { Tamaño } \\
\text { partícula }\end{array}$ & dureza & $\mathrm{P}^{*}$ \\
\hline $\begin{array}{l}\text { Aleaciones de } \\
\text { alta } \\
\text { temperaratura }\end{array}$ & Muy alta & Fina & Media & Alta \\
\hline $\begin{array}{l}\text { Fundiciones de } \\
\text { oro de alta } \\
\text { calidad }\end{array}$ & Alta & fina & Media & Alta \\
\hline $\begin{array}{l}\text { Gran volumen } \\
\text { de bronce }\end{array}$ & Medio & $\begin{array}{l}\text { Medio/ } \\
\text { grueso }\end{array}$ & medio & Medio \\
\hline $\begin{array}{l}\text { Fundiciones de } \\
\text { estatuas }\end{array}$ & Alta & Medio & Alta & Medio \\
\hline
\end{tabular}

$\mathrm{P}^{*}$ : permeabilidad.

Complementaria al método de la cera perdida, en los últimos años, se viene aplicando una nueva técnica, que es el pre-engaste de la gema, en ella la piedra natural o sintética se coloca en la cera y entra 
al proceso de tratamiento térmico del revestimiento y la colada.

El uso de esta técnica genera ventajas como reducciones de costo en la fabricación de las joyas pues las gemas se colocan en la cera y no en la pieza fundida. Se pueden ubicar y fijar en el lugar indicado con mayor precisión y no se necesita trabajo posterior de engaste.

Aunque el proceso tiene amplias ventajas, sólo se ha podido implementar con piedras sintéticas como el cuarzo y algunas naturales como el diamante, zafiro y rubí, por su estabilidad térmica [33].

Existen herramientas que permite simular el proceso de colada, el flujo de metal fundido $y$ optimizar el diseño de la pieza teniendo como base las características termo físicas de los diferentes materiales que intervienen en el proceso de fundición, con el fin de minimizar los costos de fabricación. Estas herramientas son los softwares de simulación SOLIDCAST, FLOWCAST y OPTICAST entre otros.

\subsubsection{Acabado superficial}

El acabado superficial es el proceso más importante en la pieza terminada pues permite que la superficie posea el brillo propio de las joyas.

Generalmente la forma común de realizar el proceso es mediante limpieza mecánica en húmedo, utilizando un recipiente en rotación que contiene una combinación de diferentes abrasivos entre cerámicos, plásticos y metálicos [34]. El pulido también se puede realizar de forma manual, empleando el medio abrasivo adecuado.

Para realizar un óptimo proceso, se debe tener en cuenta la reflectividad de la superficie, los mecanismos de pulido, la rugosidad y el tiempo utilizado, con el fin de disminuir las pérdidas de material y con esto los costos de fabricación [35].

Otra forma de lograr una pieza de joyería con brillo, es utilizando el proceso de electroformado [36]. En este el material empleado no es una aleación de oro, sino una de bajo costo a la cual se le aplica una capa de aleación de oro de 18, 14 o 10 quilates, con ello, el acabado superficial es igual al de una pieza del quilate mencionado pero con un costo de fabricación mucho menor [37].

\section{MÉTODOS DE EVALUACIÓN Y CARACTERIZACIÓN}

Es esencial disponer de técnicas que permitan evaluar las características y conocer el comportamiento de las aleaciones de joyería. Con frecuencia se evalúan propiedades químicas y físicas.

\subsection{Propiedades químicas}

Para las aleaciones preciosas base oro, se evalúa su composición química y resistencia a la corrosión.

En cuanto a la composición química, se han presentado modificaciones en las técnicas utilizadas, a través de los años acordes con los avances tecnológicos. En la Tabla 4 se indican algunas de las técnicas antiguas y nuevas usadas [38].

Dentro de las técnicas señaladas se encuentran una muy antigua llamada ensayo a fuego, la cual es usada para determinar la cantidad de oro y plata presente en una aleación. Adicionalmente, se presenta otra técnica más reciente como es la espectroscopia, en la cual por medio del estudio de la emisión y absorción de energía se determina la composición elemental de la aleación.

La información obtenida sobre la composición de la aleación, es determinante para contralar y ajustar el proceso de fabricación, así como garantizar el quilate de la misma al comprador.

Por otra parte, la resistencia a la corrosión es un aspecto a considerar dentro de la evaluación química de estas aleaciones, debido a que con esta se valora su respuesta a los diferentes medios.

TABLA 4

Técnicas usadas para la caracterización de las aleaciones [38]

\begin{tabular}{|c|c|c|}
\hline \multirow{4}{*}{ Antiguo } & Técnica & destructiva \\
\hline & \multirow{2}{*}{$\begin{array}{l}\text { Ensayo a fuego } \\
\text { Densidad (principio de }\end{array}$} & $\mathrm{Si}$ \\
\hline & & No \\
\hline & raya & Si \\
\hline \multirow{3}{*}{ Moderno } & $\begin{array}{l}\text { Difracción de rayos } X \\
(\mathrm{DRX}), \text { Fluorescencia de } \\
\text { rayos } X(F R X)[39]\end{array}$ & $\begin{array}{l}\text { No, } \\
\text { superficial }\end{array}$ \\
\hline & $\begin{array}{l}\text { Espectroscopia de } \\
\text { absorción atómica (AAS) }\end{array}$ & No, superficial \\
\hline & $\begin{array}{l}\text { Espectroscopia del plasma } \\
\text { (ICP) }\end{array}$ & $\begin{array}{l}\mathrm{Si} \text {, muestra en } \\
\text { solución. }\end{array}$ \\
\hline
\end{tabular}

Los tipos de corrosión más frecuentes en las aleaciones preciosas base oro, son: la corrosión bajo tensión y corrosión inter-granular. Como consecuencia se presenta fractura frágil a lo largo de los límites de grano en el material, causada por la pérdida de las propiedades mecánicas.

La Resistencia a la corrosión se puede evaluar a través de curvas de polarización potenciodinámicas, previa inmersión de la aleación en una solución electroquímica (que puede ser un ácido o una sal). Estas curvas dan información de los potenciales críticos y zonas de pasivación y actividad [40]. La velocidad de corrosión puede ser determinada mediante técnicas electroquímicas como: polarización lineal, método de extrapolación de Tafel y espectroscopia de impedancia electroquímica (EIS). 


\subsection{Propiedades físicas}

Entre las propiedades físicas más estudiadas en las aleaciones base oro se encuentran: la determinación del color, las propiedades mecánicas (resistencia y dureza), las metalúrgicas (la metalografía y la colabilidad) y las propiedades térmicas (punto de fusión y temperatura de transición vítrea).

A nivel comercial las aleaciones base oro se adquieren de acuerdo a los quilates, lo cual no es más que una forma de clasificarlas teniendo en cuenta la cantidad de oro presente. En la Tabla 5, se encuentra la relación entre la composición, color y quilate [41].

TABLA 5

Aleaciones base oro y su color dependiendo de la composición de los elementos [41]

\begin{tabular}{|c|c|c|c|c|}
\hline \multicolumn{5}{|c|}{ compusicion ue ius eiemiemios [4 I] } \\
\hline \multirow{2}{*}{ Quilate } & \multicolumn{3}{|c|}{ \% composición en peso } & \multirow{2}{*}{ Color } \\
\hline & $\mathrm{Au}$ & $\mathrm{Ag}$ & $\mathrm{Cu}$ & \\
\hline 24 & 100 & - & - & Amarillo \\
\hline 22 & 91.7 & 5.1 & 2.8 & Amarillo \\
\hline 22 & 91.7 & 3.2 & 5.1 & Amarillo oscuro \\
\hline 21 & 87.5 & 4.5 & 8.0 & Amarillo-rosado \\
\hline 21 & 87.5 & 1.75 & 10.75 & Rosado \\
\hline 21 & 87.5 & - & 12.5 & Rojo \\
\hline 18 & 75.0 & 16.0 & 9.0 & Amarillo claro \\
\hline 18 & 75.0 & 12.5 & 12.5 & Amarillo \\
\hline 18 & 75.0 & 9.0 & 16.0 & Rosado \\
\hline 18 & 75.0 & 4.5 & 20.0 & Rojo \\
\hline
\end{tabular}

Por tanto, se pueden encontrar aleaciones con diferentes porcentaje de oro 0 quilate, pero manteniendo el mismo color.

Para determinar de manera precisa su color, la simple inspección visual podría resultar en una apreciación subjetiva de éste, sin embargo, en la actualidad existen varios métodos cuantitativos que indican de forma precisa cual es el color presente en las aleaciones. Un método utilizado para caracterizar de forma objetiva el color de las aleaciones lo describe el sistema CIELAB (The International Commission on Illumination) [42].

El sistema CIELAB, expresa el color en tres coordenadas $L^{*}, a^{*} y b^{*}$. Donde $L^{*}$ es la iluminación, $a^{*}$ es la intensidad de color entre verde y rojo; $y b^{*}$ es la intensidad entre azul y amarillo, en la Fig. 8 , se puede observar un esquema de las coordenadas del sistema.

De acuerdo al sistema CIELAB, los colores se encuentran en un rango de coordenadas, por tanto, es necesario estandarizar el proceso, para ello existe la ayuda de la ASTM (American Society for Testing and Materials), la cual mediante sus normas, establecen los valores de las coordenadas $L^{*}, a^{*}, b^{*}$, para estandarizar el color de las diferentes aleaciones [43], [44].

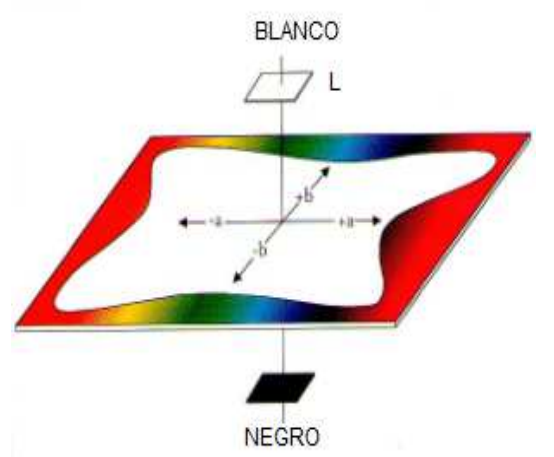

Fig. 8. Coordenadas del sistema CIELAB [42]

En cuanto a las propiedades mecánicas, las referencias señalan la resistencia a la tensión y la dureza como las pruebas mecánicas aplicables para caracterizar las aleaciones preciosas base oro. En la Tabla 6, se encuentran algunos valores de dureza y resistencia a la tensión para aleaciones base oro de diferentes quilates [45].

Se debe recordar que la ASTM, regula la forma por medio de la cual se pueden realizar los ensayos para estas pruebas [46], [47].

TABLA 6

Propiedades mecánicas de aleaciones base oro [45]

\begin{tabular}{lllll}
\hline Quilate & $\begin{array}{l}\text { Aleado } \\
\%\end{array}$ & color & $\begin{array}{l}\text { Dureza } \\
\mathrm{HV}\end{array}$ & $\begin{array}{l}\text { Tensión } \\
\mathrm{N} / \mathrm{mm}^{2}\end{array}$ \\
\hline 24 & 0 & amarillo & 20 & 124 \\
18 & $16 \mathrm{Ag}$ & & & \\
& $9 \mathrm{Cu}$ & rosa & 160 & 550 \\
14 & $9 \mathrm{Ag}$ & rojo & 160 & 550 \\
\hline
\end{tabular}

Complementaria a las pruebas indicadas está la metalografía, la cual puede evaluar la estructura presente en la fundición, y verificar si ha sido sometida a un proceso de tratamiento térmico o mecánico. Las normas ASTM ayudan a establecer la forma correcta de realizar la preparación metalográfica, el ataque más conveniente para revelar la microestructura [48] y la estimación del tamaño de grano [49]. En la Fig. 9, se muestra la microestructura de una aleación base oro de 14 quilates, la cual presenta una fuerte segregación, diferencia de concentración, dendrítica [50].

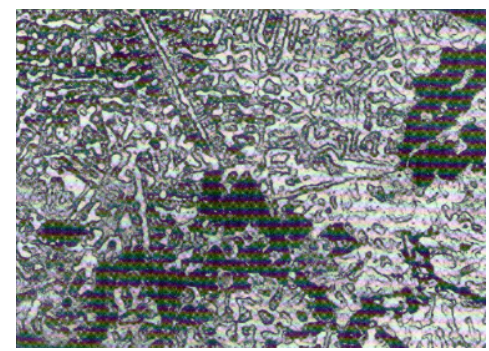

Fig. 9. Fuerte segregación dendrítica en aleación de oro de 14 quilates a $200 X$ [50] 
Otra propiedad física que es determinante en el proceso de colada de las aleaciones es la colabilidad, la cual es la capacidad que tiene de fluir un metal fundido. Esta se puede evaluar de diversas formas, sin embargo, un método muy utilizado en joyería es la fundición de una malla, con el fin de hallar el porcentaje de área llenada [51], [52] Para determinar esta propiedad se establece un factor matemático, el cual se obtiene dividiendo el área llenada sobre el área total de la malla, multiplicando por 100. En la Fig. 10 , se muestra una malla de colabilidad antes y después de ser fundida.

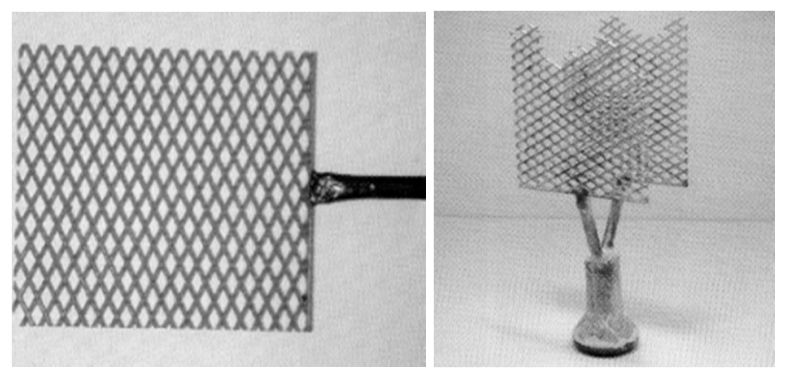

Fig. 10. Prueba de colabilidad [51]

Otro factor a considerar en el proceso de fabricación de las aleaciones es la contracción [53], la cual se presenta en el proceso de solidificación, pudiendo generar porosidad y rechupes (cavidades generadas por contracción), discontinuidades que disminuyen las propiedades del material. Por lo anterior cuando se funde se debe proveer una parte de metal líquido para compensar la contracción.

Teniendo en cuenta lo mencionado, es importante poder evitar la presencia de defectos en la fundición, con el fin de garantizar las propiedades que son necesarias para la pieza. En la Tabla 7, se encuentran algunos defectos generados por el proceso de fundición, así como algunas acciones recomendadas para minimizar su presencia.

\section{TABLA 7}

Defectos y posibles acciones correctivas (1/2)

\begin{tabular}{|c|c|c|}
\hline defecto & Posible causa & acciones \\
\hline $\begin{array}{l}\text { Burbujas, } \\
\text { esferas } \\
\text { completas } \\
\text { [54] }\end{array}$ & $\begin{array}{l}\text { Mal aplicación de vacío. } \\
\text { Ciclo de trabajo largo } \\
\text { del revestimiento. } \\
\text { Mala mezcla del } \\
\text { revestimiento. }\end{array}$ & $\begin{array}{l}\text { Realizar revisión } \\
\text { del equipo de } \\
\text { vacío. } \\
\text { Realizar la mezcla } \\
\text { de polvos a } 20-21^{\circ} \\
\text { C. }\end{array}$ \\
\hline $\begin{array}{l}\text { Burbujas, } \\
\text { Esferas } \\
\text { incompletas } \\
\text { [55] }\end{array}$ & $\begin{array}{l}\text { Superficies de tensión } \\
\text { en la superficie de la } \\
\text { cera debido a grasa, o } \\
\text { mugre. }\end{array}$ & $\begin{array}{l}\text { Considere lavar la } \\
\text { cera, o limpiar a } \\
\text { superficie. }\end{array}$ \\
\hline $\begin{array}{l}\text { Fundición } \\
\text { incompleta } \\
{[56]}\end{array}$ & $\begin{array}{l}\text { Metal o molde frio } \\
\text { durante la fundición. } \\
\text { Bebederos incorrectos. } \\
\text { Oxidación incompleta } \\
\text { de carbón. }\end{array}$ & $\begin{array}{l}\text { Incremento en la } \\
\text { temperatura de } \\
\text { colada. } \\
\text { Revisar que el } \\
\text { precalentamiento } \\
\text { del horno sea } \\
\text { adecuado. }\end{array}$ \\
\hline
\end{tabular}

TABLA 7

Defectos y posibles acciones correctivas (2/2)

\begin{tabular}{|c|c|c|}
\hline $\begin{array}{l}\text { Inclusiones } \\
\text { en la colada } \\
\text { [57] }\end{array}$ & $\begin{array}{l}\text { Diseño pobre del } \\
\text { sistema de alimentación } \\
\text { del metal. } \\
\text { Ruptura del crisol. } \\
\text { Metal contenga } \\
\text { partículas desconocidas }\end{array}$ & $\begin{array}{l}\text { Eliminar las curvas } \\
\text { cerradas en el } \\
\text { bebedero. } \\
\text { No usar crisoles } \\
\text { viejos. }\end{array}$ \\
\hline $\begin{array}{l}\text { Porosidad en } \\
\text { el metal de } \\
\text { fundición } \\
\text { [58], [59] -[60] }\end{array}$ & $\begin{array}{l}\text { Temperatura de colada } \\
\text { o del molde demasiado } \\
\text { alta. } \\
\text { Usar un metal de baja } \\
\text { calidad. }\end{array}$ & $\begin{array}{lr}\text { Usar } & \text { una } \\
\text { temperatura } & \text { de } \\
\text { fundición alta. } & \\
\text { Usar } & \text { baja } \\
\text { temperatura } & \text { del } \\
\text { molde. } & \\
\text { Usar } & \text { más } \\
\text { fundente. } & \\
\end{array}$ \\
\hline
\end{tabular}

Otra propiedad física de importancia, es la caracterización térmica, la cual aporta información importante sobre las aleaciones, pues, entre otros aspectos, el punto de fusión es determinante en el proceso de obtención de la aleación. En la tabla 8 se presentan los puntos de fusión de algunas aleaciones preciosas [61].

TABLA 8

Puntos de fusión de aleaciones de oro, en diferentes quilates [61]

\begin{tabular}{lllll}
\hline quilate & $\begin{array}{l}\text { Aleado } \\
\%\end{array}$ & color & $\begin{array}{l}\text { Densidad } \\
\mathrm{G} / \mathrm{cm}^{3}\end{array}$ & $\begin{array}{l}\text { Punto de } \\
\text { fusión }{ }^{\circ} \mathrm{C}\end{array}$ \\
\hline 23.76 & $1 \mathrm{Ti}$ & amarillo & 19 & 1090 \\
21 & $\begin{array}{l}1.75 \mathrm{Ag} \\
10.5 \mathrm{Cu}\end{array}$ & rosa & 16.8 & 952 \\
18 & $\begin{array}{l}12.5 \mathrm{Ag} \\
12.5 \mathrm{Cu}\end{array}$ & amarillo & 15.45 & 895 \\
\hline
\end{tabular}

Sumado a lo mencionado, con análisis térmico, se pueden caracterizar las aleaciones vítreas, ya que se usa la habilidad de formación del vidrio como principio para describir este tipo de aleaciones, el cual tiene varias factores que lo pueden describir dependiendo del investigador que lo propone. Uno es la relación que se muestra en la ecuación (1) [62].

$$
\Delta T_{x}=T_{x}-T_{g}
$$

Donde $\Delta T_{x}$ es el parámetro utilizado para describir la habilidad de formación de vidrios, $T_{x}$ es la temperatura a la cual comienza a cristalizar y $T_{g}$ es la temperatura de transición vítrea.

Para el investigador Lu y Liu [63] el factor que describe la habilidad de formación del vidrio está dado por la ecuación (2).

$$
\gamma=\frac{T_{x}}{T_{g}+T_{l}}
$$

Donde $\gamma$ es la habilidad de formación del vidrio y $T_{L}$ es la temperatura de líquido.

Las variables mencionadas se pueden determinar mediante la calorimetría diferencial de barrido, un análisis térmico donde se mide la diferencia de calor 
en función de la temperatura de una muestra. Una técnica alternativa es el análisis térmico diferencial, en este se miden las transiciones endotérmicas y exotérmicas en función de la temperatura. Las dos técnicas mencionadas se complementan con la termogravimetría en la cual se muestra la diferencia de masa con el aumento de temperatura.

En la Fig. 11, se muestran los resultados de realizar la calorimetría diferencial de barrido a una aleación base de Ag-Mg-Ca, en ella se puede observar como el cambio de composición de los elementos constituyentes producen una disminución en la temperatura de fusión, así como modificaciones en las temperaturas de transición vítrea y temperatura de inicio de la cristalización.

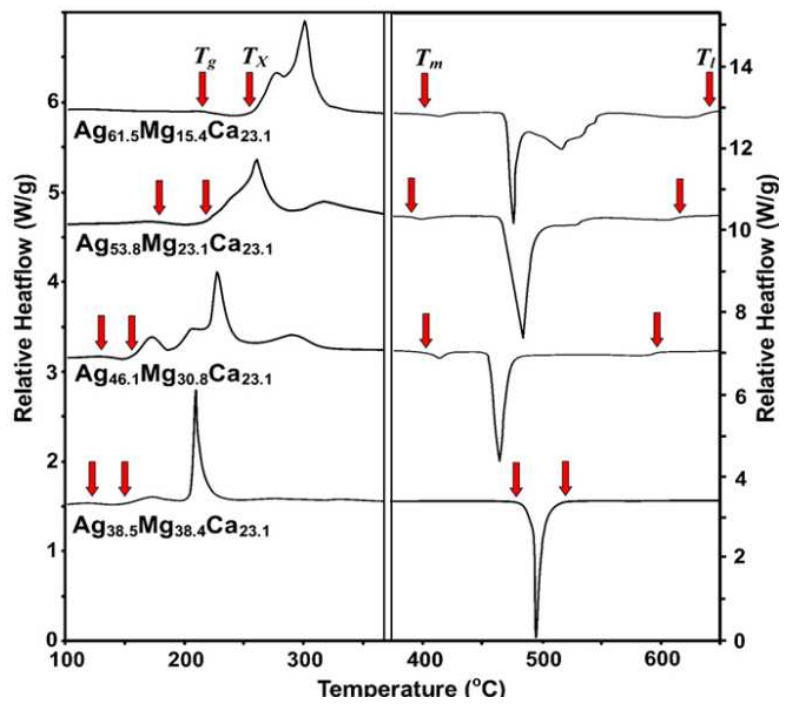

Fig. 11 Calorimetría diferencial de barrido aplicado al vidrio metálico $\mathrm{Ag}-\mathrm{Mg}$-Ca a diferentes composiciones [64]

\section{TRABAJOS FUTUROS}

La revisión bibliográfica realizada permitió establecer dos posibles trabajos futuros de interés para la industria de la joyería:

- Diseño y fabricación de aleaciones preciosas con bajos puntos de fusión, con el fin de minimizar los choques térmicos entre aleación-gema y poder implementar el proceso de microfundición con gemas naturales pre-engastadas, como el caso de la esmeralda colombiana.

- Establecimiento del proceso de fabricación de aleaciones preciosas no cristalinas, teniendo en cuenta sus excelentes propiedades tanto químicas como físicas, con el fin de implementarlas en la joyería.

Estos estudios constituyen retos tanto para la industria como para la investigación en el campo de las aleaciones preciosas.

\section{CONCLUSIONES}

Uno de los factores más importantes en el momento de la fabricación de una aleación es la selección adecuada los elementos de aleación, ya que de ello depende que las propiedades físicas y químicas cumplan los requisitos de uso.

Una ayuda determinante para establecer la composición más adecuada de una aleación son los diagramas de fase, ya que indican de manera aproximada las fases presentes en una composición determinada, y con ello se puede predecir las propiedades en esa mezcla, así como los posibles colores de la aleación.

Determinar las condiciones óptimas de fundición, con la ayuda de software de simulación, permite disminuir defectos indeseados en la aleación fabricada, optimizar los diseños y reducir costos de fabricación.

La inclusión de nuevas técnicas de evaluación y caracterización de las aleaciones preciosas base oro permiten verificar con mayor precisión sus característica siendo herramientas de apoyo tanto para la investigación como en la aplicación industrial.

Los avances tecnológicos, software de simulación y el surgimiento de nuevas técnicas, permiten impulsar el desarrollo de aleaciones base oro con características predeterminadas, diseños de modelos adecuados y optimizando el proceso de fundición. Lo mencionado con el fin de minimizando costos de fabricación y garantizar la confiabilidad del producto.

\section{AGRADECIMIENTOS}

A la vicerrectoría de investigaciones y a Colciencias por la financiación del proyecto de investigación código interno 9418 y titulado: "Innovación del Método de Gemas Pre-engastadas en el Proceso de Microfundición, para su Implementación en la Joyería con Esmeraldas Colombianas".

\section{REFERENCIAS}

[1] M. F. Grimwade, "A plain Man's guide to alloy phase diagrams: their use in jewellery manufacture - part 1," World Gold Council, Issue 29, pp. 2-15, Jun 1990.

[2] D. R Askeland and P. P. Phulé. Askeland. Ciencia e ingeniería de los materiales. México: CENGAGE Learning, pág, 357-407, 2009.

[3] A. Valencia. "Metalurgia Física". Universidad de Antioquia. Pág. 430-431, Nov 1987.

[4] M. F. Grimwade, "A plain man's guide to alloy diagrams: their use in jewellery manufacture- part 2," World Gold Council, Issue 30, pp. 8-7, Sum 2000.

[5] J. J Hostynek, "Gold: An Allergen of Growing Significance. Food and Chemical Toxicology," Elsevier, pp. 839-844, Apr 1997.

[6] M. García Ramírez. "Evaluación cromática y microstructural de una aleación de oro blanco al $\mathrm{Mn}$ en estado fundido $\mathrm{y}$ tratado térmicamente". Tesis de maestría, dirigido por D. Y. 
Peña Ballesteros y A. Alonso Baquero, Universidad Industrial de Santander, Bucaramanga, Santander, pág. 1-2, dic. 2008.

[7] R. Rushforth, "Don't let niquel get under your skin- the European experience," World Gold Council, Issue 28, pp. 210 Spr 2000.

[8] M. F. Grimwade, "The $14^{\text {th }}$ Santa Fe Symposium on jewellery manufacturing technology," World Gold Council, Issue 30, pp. 23-30 Sum 2000.

[9] Guzmano G, "Influence of $\mathrm{Si}, \mathrm{Ni}$ and $\mathrm{Co}$ additions on gold alloy for investment cast process," Elsevier, pp. 252-258, 2001.

[10] F. Arbini, "Effect of cobalt additions on the properties of $5 \mathrm{~N}$ red gold alloys," World Gold Council, Issue 25, pp. 31-35 Apr 1999.

[11] J. C. Mccloskey, P. R. Welch and S. Aithal, "The effect of silicon desoxidation and grain refinement on the production performance of a 14 karat yellow gold casting alloy," World Gold Council, Issue 30, pp. 4-7, Sum 2000.

[12] G. Lockwood, "The practical gold-worked," World Gold Council, Issue 7, pp. 15-21 Nov 1877.

[13] J. Wright, "Technical manual for gold jewellery," World Gold Council, pp. 2.3, 2001.

[14] M. F. Grimwade, "Solidification of metalsm," World Gold Council, Issue 2, pp. 11-16, Jun 1990.

[15] M. F. Grimwade, "Working and annealing," World Gold Council, Issue 2, pp. 17-22, Jun 1990

[16] W. H Wang, C. Dong and C. H. Shek, "Bulk metallic glasses," Elsevier, pp, 45-89, 2004.

[17] J. Schroers and B. Lohwongwatana, "Precious Bulk Metallic Glasses for Jewelry applications," Elsevier, pp. 235-238, 2007.

[18] W.S. Rapson, "Gold refining for the jewellery producer," World Gold Council, Issue 14, pp. 2-9 Nov 1994.

[19] M. F. Grimwade, "Melting, Alloying and casting," World Gold Council, Issue 2, pp. 5-10, Jun 1990.

[20] E. Soinila, K. Antin, S. Bossuyt and Hänninen, "Bulk metallic glass tube casting," Journal of Alloys and Compounds, Elsevier, pp. 210-213, 2011.

[21] Patrick Scott. Cad Software for Jewelry Design: A Comprehensive Survey. The 19th Santa Fe Symposium on Jewelry Manufacturing. Pp. 409-422, 2005.

[22] M. Bovin and P. Bovin. "Jewellery making for school, tradesmen, craftsmen". Forest Hill, 1976.

[23] M. G. Malagoli, "CAD-CAM technology: transforming the goldsmith's workshop," World Gold Council, Issue 31, pp. 3135 Spr 2002.

[24] L. C. Molinary and M. C. Megazinni, "The role of CAD/CAM in the modern jewelery business," World Gold Council, Issue 23, pp. 3-7 Spr 2002.

[25] Schuster, Hubert; Bolzonella, Alberto. The Preparation of the master mould- The Key Point of Stone-In-Place Casting. The 19th Santa Fe Symposium on Jewelry Manufacturing. pp. 399408, 2005.

[26] J. Wright, "Technical manual for gold jewellery," World Gold Council, pp. 3.2, 3.3, 2001.

[27] L. Kellenborg"Modelling in wax for jewellery and sculpture". Forest Hill, 1981.

[28] W. Corti, Christopher. Basic Metallurgy of the Preciuos Metals. Santa Fe Symposium, pp. 77-108, 2007.

[29] C. H. Schwartz, "Chemical and physical properties of investment," Santa Fe Symposium on Jewelry Manufacturing Technology, pp. 99, 1987.

[30] J. Wright, "Technical manual for gold jewellery," World Gold Council, pp. 3.4, 2001.
[31] R. Carter, "Effects of water quality and temperature on investment casting powder," World Gold Council, Issue 32, pp. 7-17 Sum 1993.

[32] P. J, "Investment powders and investment casting," World Gold Council, Issue 28, pp. 12-17 Spr 2000.

[33] H. Schuster and A. Bolzonella. "The Preparation of the master mould- The Key Point of Stone-In-Place Casting". Santa Fe Symposium, pp. 399-408, 2005.

[34] M. Dreher, "Bulk finishing of gold jewellery- I Wet tumbling: principios básicos," World Gold Council, Issue 26, pp. 7-10 Jul 1999.

[35] S. Alviti, "Getting gold to glitter or all that glitters is gold - the mass finishing of gold jewelry," World Gold Council, Issue 26, pp. 11-15 Jul 1999.

[36] G. Raykhtsaum and D. P. Agarwal, "Breakthrough in laser marking for precious metals," World Gold Council, Issue 24, pp. 10-13 Sep 1998.

[37] Ch. J, "Electroforming of gold alloys- the ARTFORM" process," World Gold Council, Issue 26, pp. 2-15 Jul 1995.

[38] A. Marucco and W. Stankiewicz, "Development of an XRF spectrometry analytical method for gold determination in gold jewellery alloy," World Gold Council, Issue 24, pp. 14-22 Sep 1998.

[39] P. Jalas, J. P. Ruottinen and S. Hemminki, "XRF analisis of jewelry using fully standardless fundamental parameter approach," World Gold Council, Issue 35, pp. 28-34 Sum 2002

[40] S. Mozgovoy, J. Heinrich, U.E. Klotz, and R. Busch, "Investigation of mechanical, corrosion and optical properties of an 18 carat Au-Cu-Si-Ag-Pd bulk metallic glass," Elsevier, pp. 2289 - 2291, 2010.

[41] W. Corti, Christopher. Basic Metallurgy of the Preciuos Metals. Santa Fe Symposium on Jewelry Manufacturing Technology, 2007, pp. 77-108.

[42] C. Cretu and E. V. Lingen, "Colured gold alloy," World Gold Council, Issue 30, pp. 30-39 Sum 2000.

[43] American Society for Testing and Materials. "Standard Practice for Computing the Colors of Objects by Using the CIE System". ASTM E308-13, pp. 1-44, Jan 2014.

[44] American Society for Testing and Materials. "Standard Practice for Calculating Yellowness and Whiteness Indices from Instrumentally Measured Color Coordinates". ASTM E313-10, pp. 1-6, Jan 2014.

[45] M. Grimwade. "Interdisciplinary science". Inst. Of Materials. Pp. 373, 1992.

[46] American Society for Testing and Materials. "Standard Test Methods for Tension Testing of Metallic Materials". ASTM E8 /E8M - 13a, pp. 1-28, Feb 2014.

[47] American Society for Testing and Materials. "Standard Test Method for Brinell Hardness of Metallic Materials". ASTME1012, pp. 1-32, Feb 2014

[48] American Society for Testing and Materials. "Standard Guide for Preparation of Metallographic Specimens". ASTM E3-11, pp. 1-12, Feb 2014.

[49] American Society for Testing and Materials. "Standard Test Methods for Determining Average Grain Size". ASTM E11212, pp. 1-27, Feb 2014

[50] D. Ott and U. Schindler, "Metallography of gold alloys," World Gold Council, Issue 33, pp. 6-11 Win 2001.

[51] D. Zito, "Coloured carat golds for Investment casting," World Gold Council, Issue 31, pp. 35-42 Spr 2001.

[52] D. Ott, "Gold casting alloy 14 and 18 carat," World Gold Council, Issue 7, pp. 2-7 Spr 1992. 
[53] C. Creud, E. Van der Lingen and L. Glaner, "Hard 22 Carat gld alloy," World Gold Council, Issue 7, pp. 25-29 Sum 2000.

[54] L. Diamond, "Casting defects from model to finished product". Santa Fe Symposium on Jewelry Manufacturing Technology, pp. 149, 1987.

[55] C. J. Raub. "Testing quality of jewellery casting" Santa Fe Symposium on Jewelry Manufacturing Technology, pp.107, 1987.

[56] D. Ott, "Ingot casting and continuous casting," World Gold Council, Issue 13, pp. 45-48 Nov 1997.

[57] D. Ott, "Description of defects," World Gold Council, Issue 13, pp. 37-40 Nov 1997.

[58] R.T. Owen, "Castings defects control," World Gold Council, Issue 13, pp. 2-15 Jul 1994.

[59] D. Ott, "Porosity in investment casting," World Gold Council, Issue 11, pp. 15-20 Nov 1993.

[60] E. Bell, "Wax elimination, burnout oand the mold's effect on porosity in casting," World Gold Council, Issue 11, pp. 21-27 Nov 1993.

[61] J. C. Wright, "Technical manual for gold jewellery," World Gold Council, Pp. 44-58, 2000.

[62] D. V. Louzguine-Luzgin, I. Zeki, T Yamamoto, H. Kawaji, C, Sueyanarayana and A. Inoue, "Structural relaxation and crystallization processes in $\mathrm{Cu}_{55} \mathrm{Hf}_{25} \mathrm{Ti}_{15} \mathrm{Pd}_{5}$ metallic glassy alloy," Elsevier, pp. 177-181, 2012.

[63] A. Eugen,"Metallic glasses from "alchemy" to pure science: Present and future of design, processing and applications of glassy metals," Elsevier, pp. 1-39, 2012.

[64] K. laws, K. Shamlaye and M. Ferry, "Synthesis of Ag-based bulk metallic glass in the Ag-Mg-Ca-[Cu] alloy system," Elsevier, pp, 10-13, 2012. 